\title{
Modelling the AFM tip effect for quantitative analysis of precipitate volume fraction in nickel-based superalloys
}

\author{
Michel Troyon $\left({ }^{1}\right)$, Alain Hazotte $\left({ }^{2}\right)$ and Abderrahim Bourhettar $\left({ }^{1}\right)$ \\ ( $\left.{ }^{1}\right)$ Laboratoire de Microscopie Electronique, GRSM, Université de Reims, 21 rue Clément Ader, \\ 51100 Reims, France \\ $\left({ }^{2}\right)$ Laboratoire de Science et Génie des Matériaux Métalliques, Ecole des Mines de Nancy, Parc de \\ Saurupt, 54041 Nancy Cedex, France
}

(Received July 4; accepted November 16, 1994)

\begin{abstract}
Résumé. - Une méthode pour mesurer la fraction volumique des précipités dans les superalliages à base de Nickel par microscopie à force atomique est décrite. La prise en compte de la géométrie de la pointe et le développement d'un modèle théorique de l'histogramme des niveaux de profondeur dans l'image permettent de bien comprendre quel rôle les différents paramètres expérimentaux jouent et comment obtenir une bonne estimation de la fraction volumique des précipités $\gamma^{\prime}$.
\end{abstract}

\begin{abstract}
A method to measure the precipitate volume fraction in nickel-based superalloys by using atomic force microscopy is described. Taking into account the geometry of the AFM tip and modelling the depth-level histogram of the image permits a good understanding of the role played by the different experimental parameters, and allows a good estimate of the $\gamma^{\prime}$ precipitate volume fraction to be obtained.
\end{abstract}

\section{Introduction.}

In near-field microscopy the quantitative study of corrugated surfaces at the nanometer scale is hindered, because the tip is not an infinitely thin, sharp probe. The profilometry of the surface is generally not correct, the contours of the objects are dilated due to the geometry and the finite dimension of the tip, and thus the area measurements, cannot be performed with precision. This tip geometrical effect is well known by the near-field microscopists and is generally described as a tip convolution. In fact this terminology is not appropriate at all, because the tip-surface interaction is not linear. It has been recently proposed $[1,2]$ that the concepts of morphological mathematics be used to describe the image formation in near-field microscopy by a dilation, with a structuring element (the tip). If the shape of the latter is known, it is then conceivable to restore the image by performing the reverse operation (the erosion) so as to get a better image, close to the "true" object, which can be used to perform quantitative analysis. In reality, a perfectly restored image 
will never be obtainable, because certain information is completely lost during image formation: for example, the exact profile of a sharp edge cannot be obtained with a pyramidal AFM tip shape. Consequently, precise quantitative analysis cannot be done from a restored image without some prior knowledge of the true object, even if the tip shape is perfectly known.

We then show that if the structure of the object is relatively simple and approximately known (that is the case of two-phase $\gamma-\gamma^{\prime}$ Nickel-based superalloys) measurements of areas can be done with a good degree of accuracy from simple geometrical considerations on the tip shape. The volume fraction of $\gamma^{\prime}$ precipitates in $\gamma-\gamma^{\prime}$ Nickel-based superalloys is one of the parameters which is important to measure, as the mechanical properties of these materials strongly depend on it.

At the present time, the most accurate measurements of precipitate volume fraction in superalloys are available by time-consuming techniques, such as precipitate chemical extraction and weighing, or atom probe chemical analysis [3]. Scanning Electron Microscopy (SEM) associated with image analysis is currently used, but leads to systematic errors of the order of $10 \%[4,5]$. We recently proposed to use Scanning Tunneling Microscopy (STM) [6], or better Atomic Force Microscopy (AFM) [7] to measure the precipitate volume fraction with a precision of the order of 2 to $3 \%$. The basic idea of the method described in ref [7] is to image a surface chemically etched with a solution that preferentially etches $\gamma^{\prime}$ precipitates, then, using different etching times, to determine the evolution of the measured surface fraction as a function of the etching depth. The effective surface fraction, which is equal to the volume fraction [8], is then obtained by extrapolation to zero etching depth. This procedure is interesting, because at zero etching depth the tip geometry no longer affects the measurement, and thus there is no need to know the tip shape. Unfortunately, although this procedure is much shorter than the atom probe chemical analysis, it is still long. In order to get a good extrapolation, it needs to image several different specimens having different etching depths. Approximately ten images $\left(10 \times 10 \mu \mathrm{m}^{2}\right.$ size $)$ of different zones for each specimen are required for sufficient statistics to be obtained.

Here, we propose and describe a faster method of volume fraction measurement that takes into account the geometry of the tip. This permits the analysis time to be reduced by a factor of about 5 in imaging different zones of only one specimen.

\section{Experiment.}

The type of material studied here is an industrial Nickel-based superalloy, MC2, whose chemical composition is (wt \%) $8 \mathrm{Cr}-8 \mathrm{~W}-6 \mathrm{Ta}-5 \mathrm{Al}-5 \mathrm{Co}-1.5 \mathrm{Ti}-\mathrm{Ni}$ balance [9]. A MC2 single crystal bar was directionaly solidified along the $\langle 001\rangle$ crystal direction and then submitted to a standard optimization heat treatment followed by two precipitation sequences. (The precipitation heat treatment led to an homogeneous distribution of cuboidal $\gamma^{\prime}$ precipitates, with their faces parallel to the $\{001\}$ crystallographic planes. Their size is tightly distributed around about $0.5 \mu \mathrm{m}$, and the alloy chemical composition is designed to obtain about $70 \%$ of $\gamma^{\prime}$ phase [9]. Slices of about $2 \mathrm{~mm}$ thick were cut perpendicular to the bar axis with their faces parallel to a $\{100\}$ crystal plane. Then, after mechanical polishing, specimens were chemically etched with a solution known to preferentially etch $\gamma^{\prime}$ phase. The etching depth was chosen to be approximately in the range of $50 \mathrm{~nm}$. The chemical etchant used in the case of these alloys is a solution of $5 \mathrm{Occ} \mathrm{HCl}, 25 \mathrm{cc}$ $\mathrm{HNO}_{3}$ and $2 \mathrm{~g} \mathrm{CuCl}_{2}$ in $200 \mathrm{cc} \mathrm{H}_{2} 0$.

In this study the etched surfaces were observed in air by means of a commercial AFM (Nanoscope II of Digital Instrument). Measurements of etching depths were obtained from the constructor $Z$ calibration, considering that this one is reliable. A set of images related to ten different zones were used to make measurements. Images were $10 \times 10 \mu \mathrm{m}^{2}$ in size, with $400 \times 400$ pix- 
els and obtained with an $X$ scanning frequency of about $1 \mathrm{~Hz}$. The images were processed to transform a sloped surface into a flat one by least-mean-squares fit through all data points of the surface. The AFM tips used were classic $\mathrm{Si}_{3} \mathrm{~N}_{4}$ tips of pyramidal shape.

\section{Model and method.}

Figure 1 shows an AFM image of MC2 superalloy with its depth-level histogram. The two modes relative to $\gamma$ and $\gamma^{\prime}$ phases are very well differentiated. The etching depth $e_{\mathrm{m}}$ of $\gamma^{\prime}$ phase is directly measured as the distance between the two maxima of the depth-level histogram. The volume fraction of $\gamma^{\prime}$ phase can be estimated from the area fraction measurement of the $\gamma^{\prime}$ phase. Indeed, it must be remembered that the area fraction $A_{\mathrm{A}}(X)$ of a given phase $X$ on a surface intersecting a poly-phased material is a non-biased estimator of its true volume fraction $V_{\mathrm{v}}(X)$ [8]. The problem is thus to correctly separate the pixels belonging to the $\gamma^{\prime}$ precipitates from those belonging to the $\gamma$ matrix.

In reality the histogram does not only contain two modes corresponding to the two peaks, but also a population of $\gamma^{\prime}$ precipitates, the etching depth of which is smaller than the maximum etching depth $e_{\mathrm{m}}$.

Indeed, when sectioning the material, a significant number of $\gamma^{\prime}$ precipitates have a depth $e_{i}$ (below section plane) smaller than $e_{\mathrm{m}}$ (Fig. 2a) and consequently they are not etched to the maximum depth $e_{\mathrm{m}}$. A perfect specimen, probed with a perfect instrument equipped with an infinitly thin tip, would give the histogram of figure $2 \mathrm{~b}$ : a population of pixels belonging to the $\gamma$ matrix, a population of pixels belonging to the $\gamma^{\prime}$ precipitates, fully-etched at the depth $e_{\mathrm{m}}$ (the left peak in Fig. 2b) and a population of pixels belonging to non-fully etched precipitates whose depths are between zero and $e_{\mathrm{m}}$. Taking into account that the probability of cutting a cubic precipitate of size $a$ at depth $h(o<h<a)$ is uniform and can be written as:

$$
P(h, h+d h)=(1 / a) \cdot d h
$$

the $\gamma^{\prime}$ precipitate fraction area $A_{\mathrm{A}}\left(\gamma^{\prime}\right)$ can be written as the sum of two terms:

$$
A_{\mathrm{A}}\left(\gamma^{\prime}\right)=\left(1-e_{\mathrm{m}} / a\right) A_{\mathrm{A}}\left(\gamma^{\prime}\right)+\left(e_{\mathrm{m}} / a\right) A_{\mathrm{A}}\left(\gamma^{\prime}\right)
$$

The first term is related to the fraction of $\gamma^{\prime}$ precipitates having the maximum etching depth $e_{\mathrm{m}}$ (the $\gamma^{\prime}$ peak). The second one corresponds to the fraction of precipitates, the etching depth of which ranges between zero and $e_{\mathrm{m}}$. As a consequence of the equiprobability of the cutting plane section, this second population is uniformly distributed on the intermediates classes of the histogram.

However, the histogram of figure 2 is obviously not realistic ,since the AFM tip has a finite dimension which results in a dilation effect on the borders of the precipitates. The resulting alteration of the histogram can be calculated if the tip geometry is known and if one assume that all the precipitates have the same size $a$. Figure 3 schematizes an AFM tip in contact with an etched precipitate. The AFM tip can be represented [10] as a square section pyramid (semi-angle $\alpha$ ) with a spherical extremity (radius $r$ ).

Let us consider a $\gamma^{\prime}$ precipitate etched up to a depth $h\left(o<h<e_{\mathrm{m}}\right)$. As illustrated in figure 3, the precipitate area actually "seen" at deph $h$ by the tip, that is to say the area of the precipitate bottom, will be $(a-x)^{2}$, rather than $a^{2} . x$ is a function of $h$, and of the parameters $\alpha$ and $r$ defining the tip shape. It can take two different expressions:

* if $o<h<L$ only the spherical tip extremity simultaneously touches the precipitate bottom and its edge. $x$ can be written as:

$$
x=[h(2 r-h)]^{1 / 2}=r \sin \theta
$$



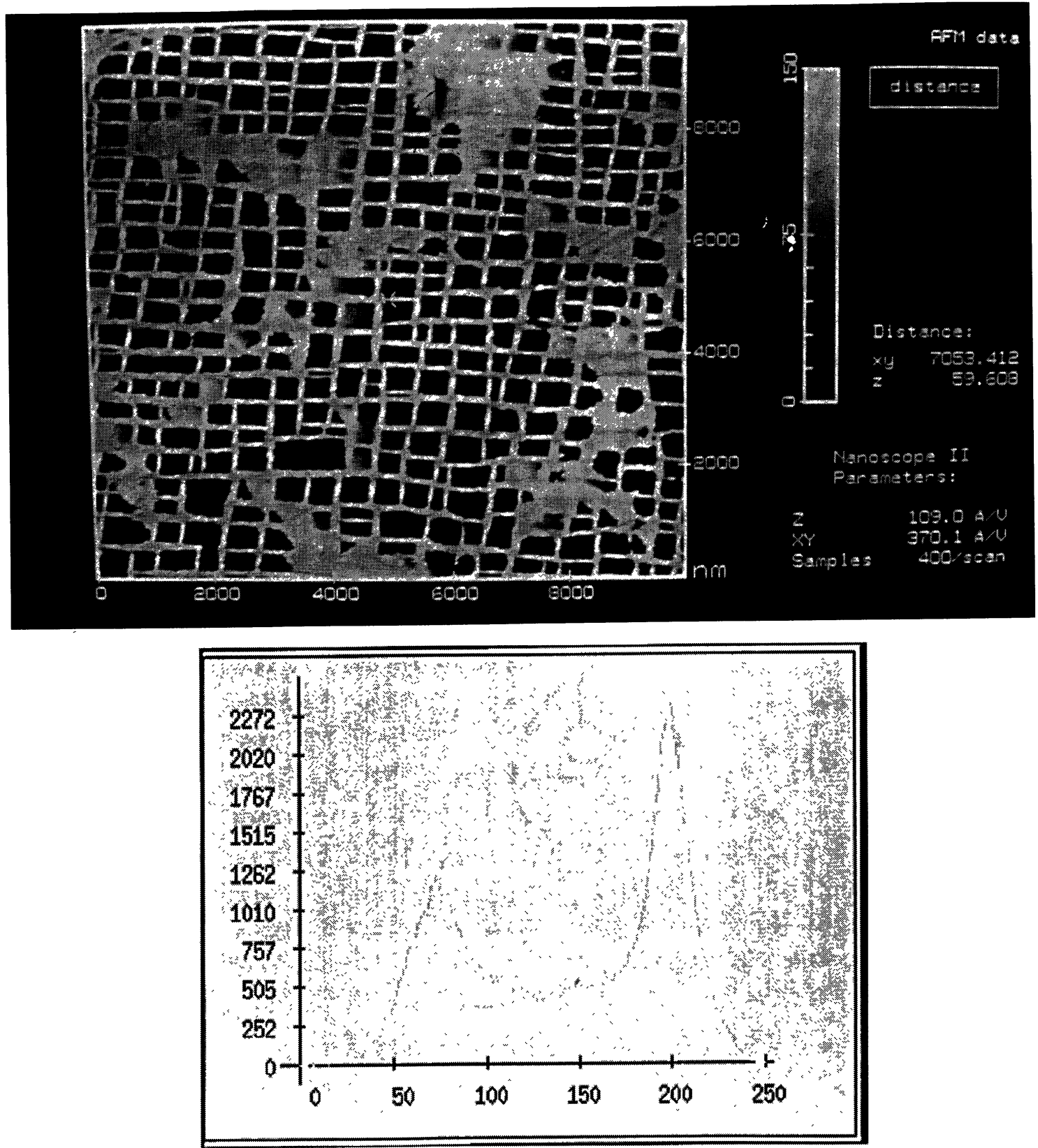

Fig. 1. - a) AFM image $\left(10 \times 10 \mu \mathrm{m}^{2}\right)$ of a $\gamma-\gamma^{\prime}$ nickel-based superalloy; b) corresponding depth-level histogram.

where $\theta$ defines the angle corresponding to the contact point between the spherical extremity and the edge of the precipitate. The maximum value is $\theta=(\pi / 2)-\alpha$ when $h=L=r(1-\sin \alpha)$.

${ }^{*}$ if $L<h<e_{\mathrm{m}}$ (case of Fig. 3), $x$ can be written as:

$$
x=(h+d) \operatorname{tg} \alpha
$$




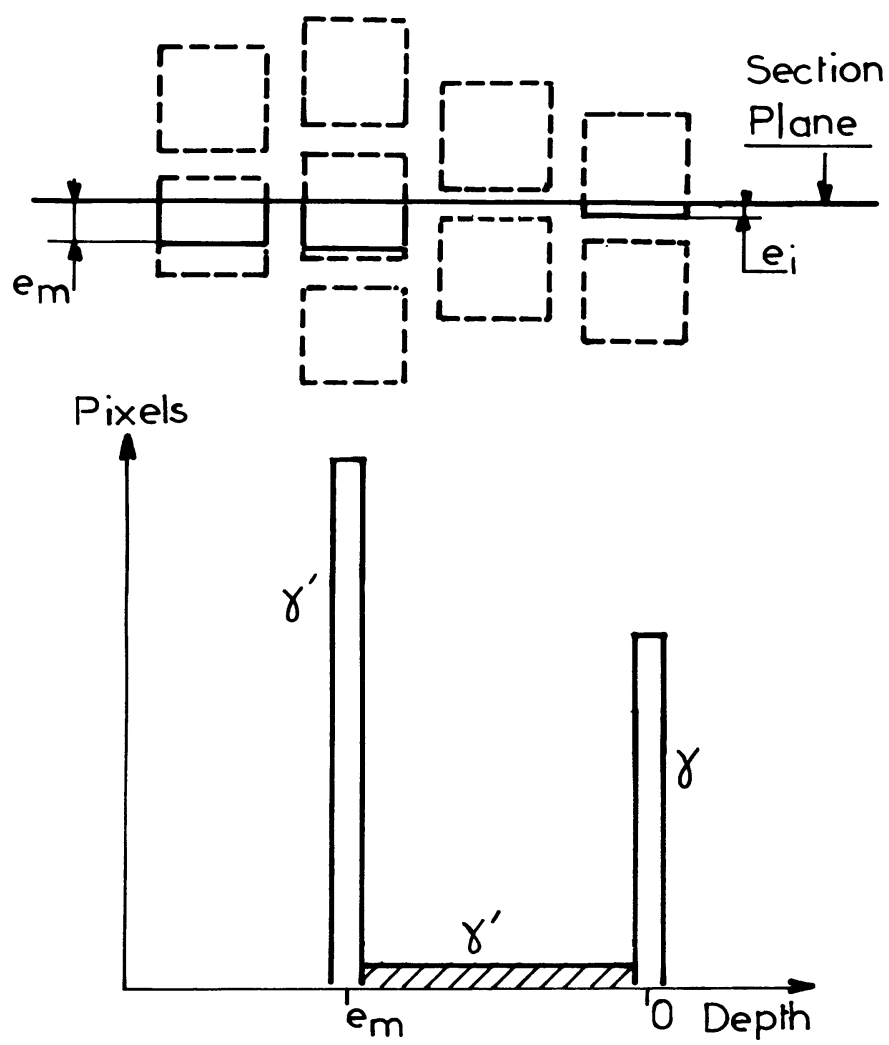

Fig. 2. - a) Schematic representation of the cubic $\gamma^{\prime}$ precipitates in the $\gamma$ matrix, with a section plane defined at random: after etching, some precipitates have a depth $e_{i}$ of between zero and the maximum depth $\left.e_{\mathrm{m}} ; \mathrm{b}\right)$ theoretical depth-level histogram that would be obtained with an infinitely thin tip and in the absence of noise.

with

$$
d=r[(1 / \sin \alpha)-1]
$$

As the tip dilation effect results in an apparent decrease in the precipitate area, this leads to a redistribution of the area corresponding to the dilated borders on the lower depths. More precisely, the part of precipitate area attributed to the depths ranging between $h$ and $h+d h$ is given by:

$$
A\left(\gamma_{h}^{\prime}\right)=[a-2 x(h)]^{2}-[a-2 x(h+d h)]^{2}
$$

In terms of a depth-level histogram, it means that the precipitate area attributed to a given depth $h\left(o<h<e_{\mathrm{m}}\right)$ results from two contributions:

- the area of the precipitate bottom (the center) etched up to $h$.

- the part of the borders (at depth $h$ ) of the precipitates etched up to depths greater than $h$.

This leads to an altered histogram, as illustrated in figure 4. The tip dilation effect causes a reduction of the $\gamma^{\prime}$ peak, most of the area redistribution benefitting at the lower depth levels. The $\gamma$ peak is not modified.

The area of the differents contributions can be calculated by taking into account the probability of obtaining each population. The total area $A_{\mathrm{T}}$ of an image, if one supposes that there is a number 


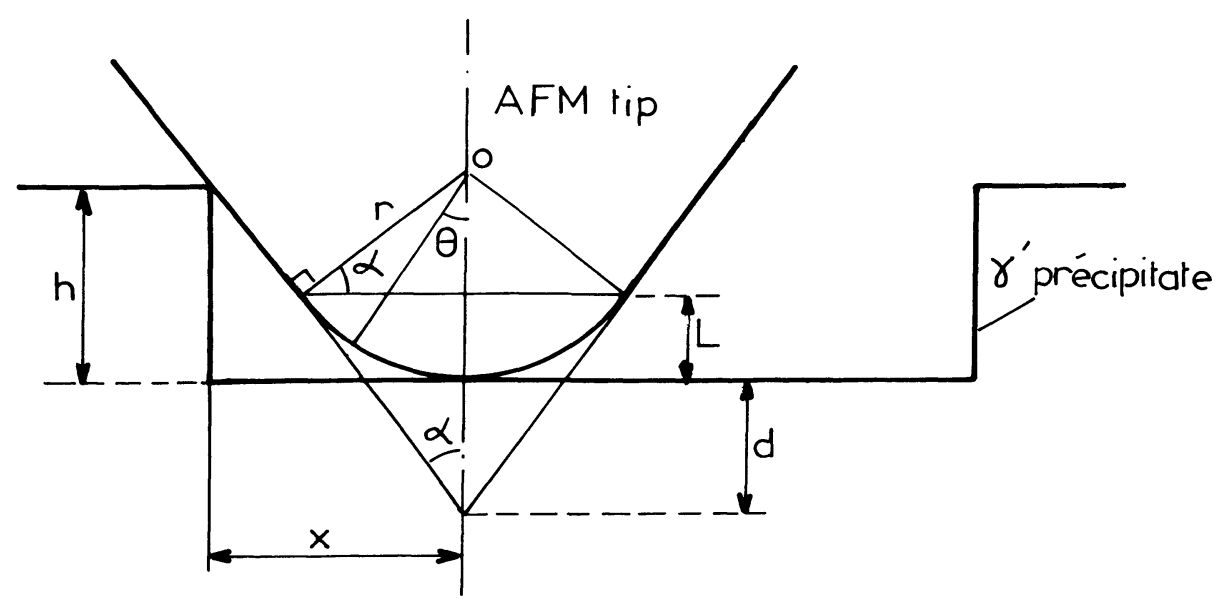

Fig. 3. - Representation of a pyramidal AFM tip with a spherical extremity in contact with the bottom of a $\gamma^{\prime}$ precipitate by its end and the side of the pyramid.

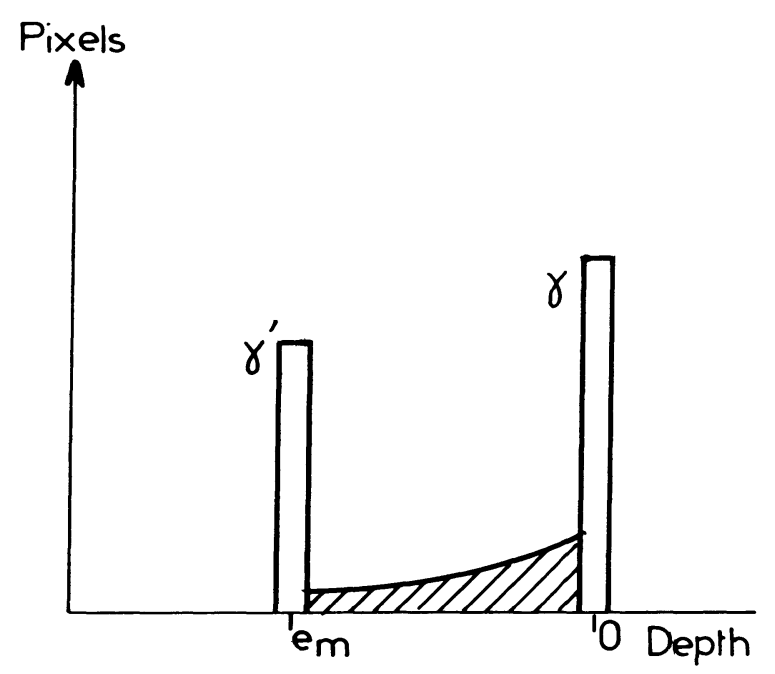

Fig. 4. - Theoretical depth-level histogram that would be obtained with the AFM tip model of figure 3 in the absence of noise.

$N$ of precipitates all identical in this image, can be written as:

$$
A_{\mathrm{T}}=A(\gamma)+N\left(A_{1}+A_{2}+A_{3}\right)
$$

The term $A(\gamma)$ is the total area of the $\gamma$ matrix. The term $A_{1}$ is the bottom area of a precipitate having the maximum etching depth $e_{\mathrm{m}}$. The proportion of this kind of precipitate is $\left(1-e_{\mathrm{m}} / a\right)$.

$$
A_{1}=\left(1-e_{\mathrm{m}} / a\right)\left[a-2 x\left(e_{\mathrm{m}}\right)\right]^{2}
$$

The term $A_{2}$ is the bottom area of a precipitate with $h<e_{\mathrm{m}}$. Taking into account the probability 
to obtain this population, $A_{2}$ can be written as:

$$
A_{2}=\int_{0}^{e_{\mathrm{m}}}(1 / a)[a-2 x(h)]^{2} \mathrm{~d} h
$$

the integral separates in two parts when $L<e_{\mathrm{m}}$, and $A_{2}$ becomes:

$$
A_{2}=\int_{0}^{\pi / 2-\alpha}(1 / a)(a-2 r \sin \theta)^{2} r \sin \theta \mathrm{d} \theta+\int_{L}^{e_{\mathrm{m}}}(1 / a)[a-2(h+d) \operatorname{tg} \alpha]^{2} \mathrm{~d} h
$$

and the term $A_{3}$ is the area corresponding to the dilated borders. The analysis of the histogram enables to measure the fraction of pixels contained in the peak $\gamma^{\prime}$, all situated at the depth $e_{\mathrm{m}}$. This area fraction $A_{\mathrm{A}}\left(\gamma^{\prime} e_{\mathrm{m}}\right)$ is equal to:

$$
A_{\mathrm{A}}\left(\gamma^{\prime} e_{\mathrm{m}}\right)=N A_{1} / A_{\mathrm{T}}=\left(N / A_{\mathrm{T}}\right)\left(1-e_{\mathrm{m}} / a\right)\left[a-2 x\left(e_{\mathrm{m}}\right)\right]^{2}
$$

Experimentally the analysis of the histogram gives us the depth $e_{\mathrm{m}}$ and the image gives us the number $N$ of $\gamma^{\prime}$ precipitates. So, if the tip shape is supposed to be known, and if one is able to measure on the histogram the area fraction $A_{\mathrm{A}}\left(\gamma^{\prime} e_{\mathrm{m}}\right)$ corresponding to the peak $\gamma^{\prime}$ the problem is solved. From the above formula the value of the precipitate size a is calculated and thus the precipitate fraction $A_{\mathrm{A}}\left(\gamma^{\prime}\right)$ is:

$$
A_{\mathrm{A}}\left(\gamma^{\prime}\right)=N a^{2} / A_{\mathrm{T}}
$$

The histogram of figure 4 is still not realistic for the reason that the experimental noise has not been taken into account. The noise can have different origins: the plateaus of the $\gamma$ matrix and the bottom of $\gamma^{\prime}$ precipitates are not perfectly flat, but show fluctuations (polishing scratches, inhomogeneities of etching, inperfect plane cube faces...); the instrument has some mechanical or electrical instabilities etc. A depth-level histogram model closer to the experience can be generated by considering a gaussian noise. This has been done assuming a similar noise for the two phases. Figure 5 shows two histograms calculated with two levels of experimental noise (standard deviations $\sigma=4$ and $10 \mathrm{~nm}$ ). The contributions of the three populations previously discussed are represented by dotted lines: the $\gamma$ matrix (right peak), the center of the precipitates etched up to $e_{\mathrm{m}}$ (left peak) and the population of non-fully etched precipitates or/and affected borders (intermediate basic line). The total histogram (sum of the three contributions) is represented by an unbroken line. One can first remark that the areas below $\gamma$ and $\gamma^{\prime}$ peaks are very different from the "true" areas (areas below the dotted lines) when the noise is high ( $\sigma=10 \mathrm{~nm})$. However, when the noise is low $(\sigma=4 \mathrm{~nm})$, the area of the $\gamma^{\prime}$ peak is close to the true value $A_{\mathrm{A}}\left(\gamma^{\prime} e_{\mathrm{m}}\right)$, whereas there is still an overestimation of the $\gamma$ peak area. A part of the pixels of the $\gamma^{\prime}$ precipitates $\left(h<e_{\mathrm{m}}\right)$ and their borders contribute to the $\gamma$ peak. This is also true for the $\gamma^{\prime}$ peak, but to a lesser extent.

One can also remark that the measurement of the maximum depth $e_{\mathrm{m}}$ from the histogram (distance between both peaks) is obtained with a very good precision (1.8\%) at low noise ( $\sigma=4$ $\mathrm{nm}$ ). This slightly deteriorates when noise increases (error of $5.4 \%$ for $\sigma=10 \mathrm{~nm}$ ). The histogram model also reveals that the two peaks are unsymmetrical due to the pixels of the intermediate population, which mainly increase the right part and the left part of the $\gamma^{\prime}$ and $\gamma$ peaks, respectively. Consequently, we propose to obtain an approximated area value of the true $\gamma^{\prime}$ peak by taking, as a measurement criteria, the double of the left part of the $\gamma^{\prime}$ peak. Under these conditions, if the noise is not important, and it seems this is the case with our experimental histograms, it should be possible to obtain an experimental $\gamma^{\prime}$ volume fraction which is only slightly overestimated.

When calculating the surface $A_{1}$ we supposed that the sides of the square section of the pyramid were parallel to the precipitate sides (Fig. 3). This means the tip scanning movement was parallel 

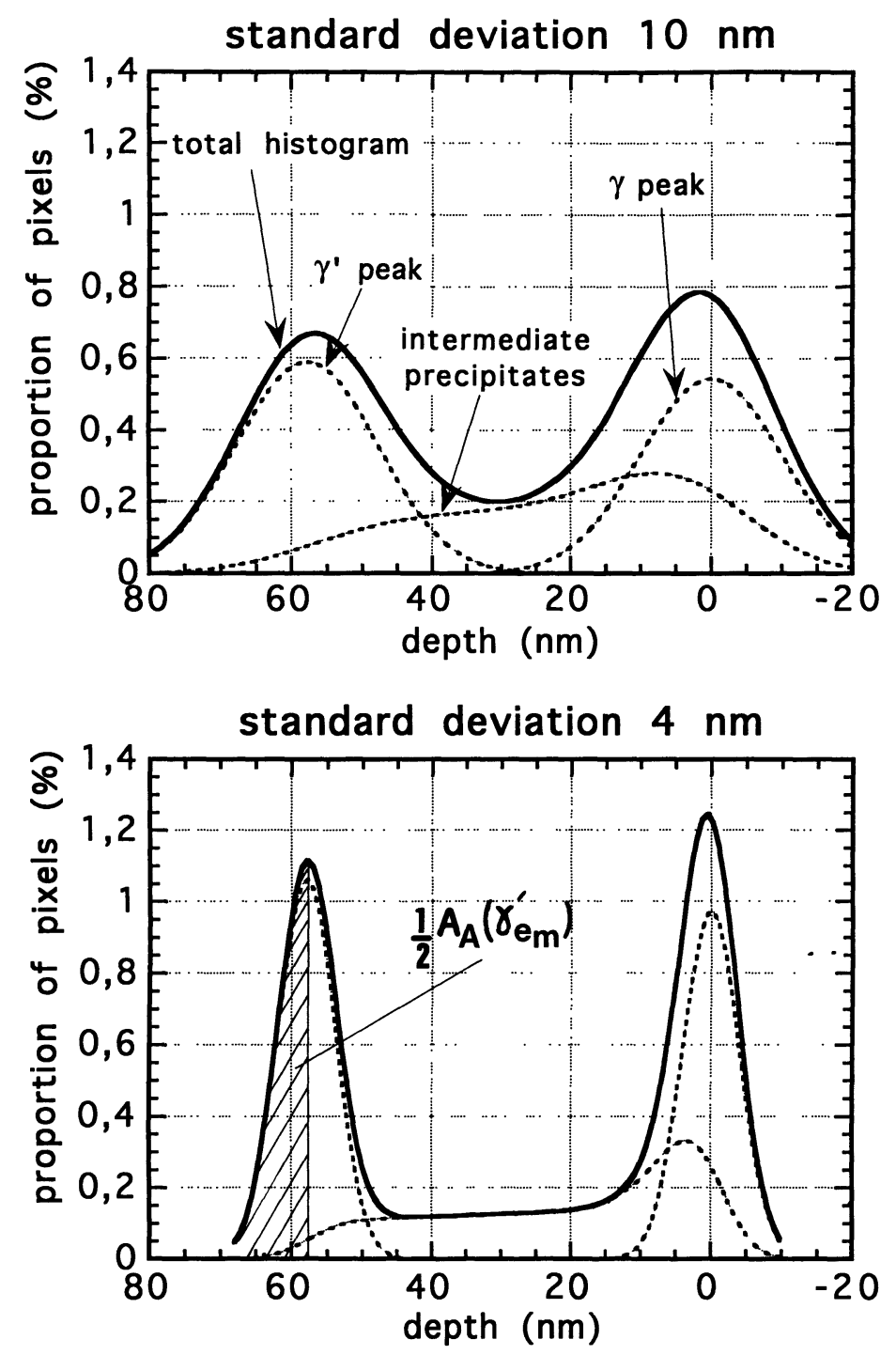

Fig. 5. - Theoretical histograms that would be obtained in presence of noise and with the tip model of figure 3. They are calculated for a precipitate size $a=500 \mathrm{~nm}$, a maximum etching depth $e_{\mathrm{m}}=58 \mathrm{~nm}$, $\alpha=36^{\circ}$ and $r=40 \mathrm{~nm}$ ). a) the noise is taken into account with a standard deviation $\sigma=10 \mathrm{~nm}$. b) $\sigma=4$ nm.

to the precipitates sides. In fact, the contact point at the tip extremity is no longer at the distance $x$ from the precipitate side, but at the distance $x^{\prime}$ (see Fig. 6) if the scanning direction is not parallel to the precipitate side. $x^{\prime}$ can be written as:

$$
x^{\prime}=x(\cos \phi+\sin \phi)
$$

where $\phi$ is the angle between the scanning direction and the precipitate side. This situation only occurs when $L<e_{\mathrm{m}}$ and thus the surface $A_{1}$ has to be corrected as:

$$
A_{1}=\left(1-e_{\mathrm{m}} / a\right)\left[a-2(\cos \phi+\sin \phi)\left(e_{\mathrm{m}}+d\right) \operatorname{tg} \alpha\right]^{2}
$$




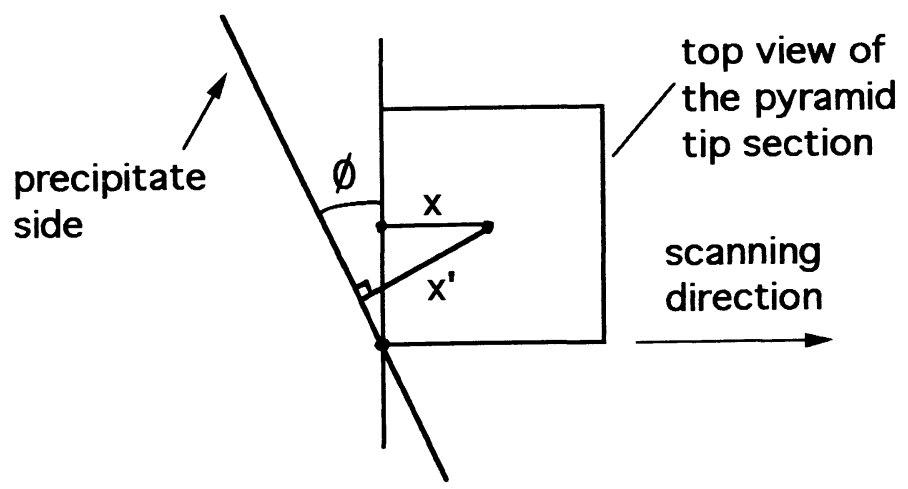

Fig. 6. - Top view of a square section of the AFM tip scanning the precipitate in a direction that is not parallel to the precipitate side. The distance between the precipitate side and the tip extremity increases to the value $x^{\prime}=x(\cos \phi+\sin \phi)$.

When $L>e_{\mathrm{m}}$ only the spherical extremity of the tip is in contact with the precipitates. So $x^{\prime}=x$ and $A_{1}$ takes the following value:

$$
A_{1}=\left(1-e_{\mathrm{m}} / a\right)\left[a-2 \sqrt{e_{\mathrm{m}}\left(2 r-e_{\mathrm{m}}\right)}\right]^{2}
$$

\section{Results and discussion.}

Let us see now what are the experimental results obtained when applying the method described above for three specimens etched at three different depths. The mean number of precipitates contained in one $10 \times 10 \mu \mathrm{m}^{2}$ image was obtained by counting the number of precipitates in ten different images; its value is $N=298$. Table I gives the experimental $\gamma^{\prime}$ area fraction values (area fraction $=$ volume fraction) calculated for three different tip radius $r=30,40,50 \mathrm{~nm}$.

Table I. - Values of the area fraction $A_{\mathrm{A}}\left(\gamma^{\prime}\right)$ calculated for three maximum etching depts $e_{\mathrm{m}}$ and three tip radius $r$. The measured area fraction $A_{\mathrm{A}}\left(\gamma^{\prime} e_{\mathrm{m}}\right)$ corresponding to the $\gamma^{\prime}$ peak is also given.

\begin{tabular}{|c|c|c|c|c|}
\cline { 2 - 4 } \multicolumn{1}{c|}{} & $e_{\mathrm{m}}(\mathrm{nm})$ & $14.9 \pm 0.7$ & $32.4 \pm 0.9$ & $58 \pm 2$ \\
\hline $\begin{array}{c}A_{\mathrm{A}}\left(\gamma^{\prime} e_{\mathrm{m}}\right) \\
(\%)\end{array}$ & & $52.7 \pm 2.1$ & $46.1 \pm 2.5$ & $35.4 \pm 1.3$ \\
\hline & & & & \\
\hline$A_{\mathrm{A}}\left(\gamma^{\prime}\right)$ & 40 & 71.4 & 70.1 & 69.2 \\
$(\%)$ & 50 & 74.3 & 73.8 & 72.1 \\
\hline
\end{tabular}

Evidently, as one might suppose, the precision of the area fraction measured by this method directly depends on the precision with which the tip shape is known. The tip half-angle $\alpha$ is well 
defined because AFM tips of pyramidal shape are produced in a highly reproductible way, originating from the semiconductor device fabrication technology. On the other hand, the tip radius may vary a little from one tip to another. The values that are generally reported in the literature are $\alpha=36^{\circ}$ and $r \sim 30$ to $40 \mathrm{~nm}$ [10]. In order to confirm these values we imaged a few tips chosen randomly at high magnification by transmission electron microscopy. Our observations effectively confirmed these published values; there is no discrepancy with $\alpha$ and the radius indeed varies around 30 to $40 \mathrm{~nm}$. Figure 7 is an example of an AFM tip extremity with $\alpha=36^{\circ}$ and $r \sim 40 \mathrm{~nm}$, imaged in transmission electron microscopy.

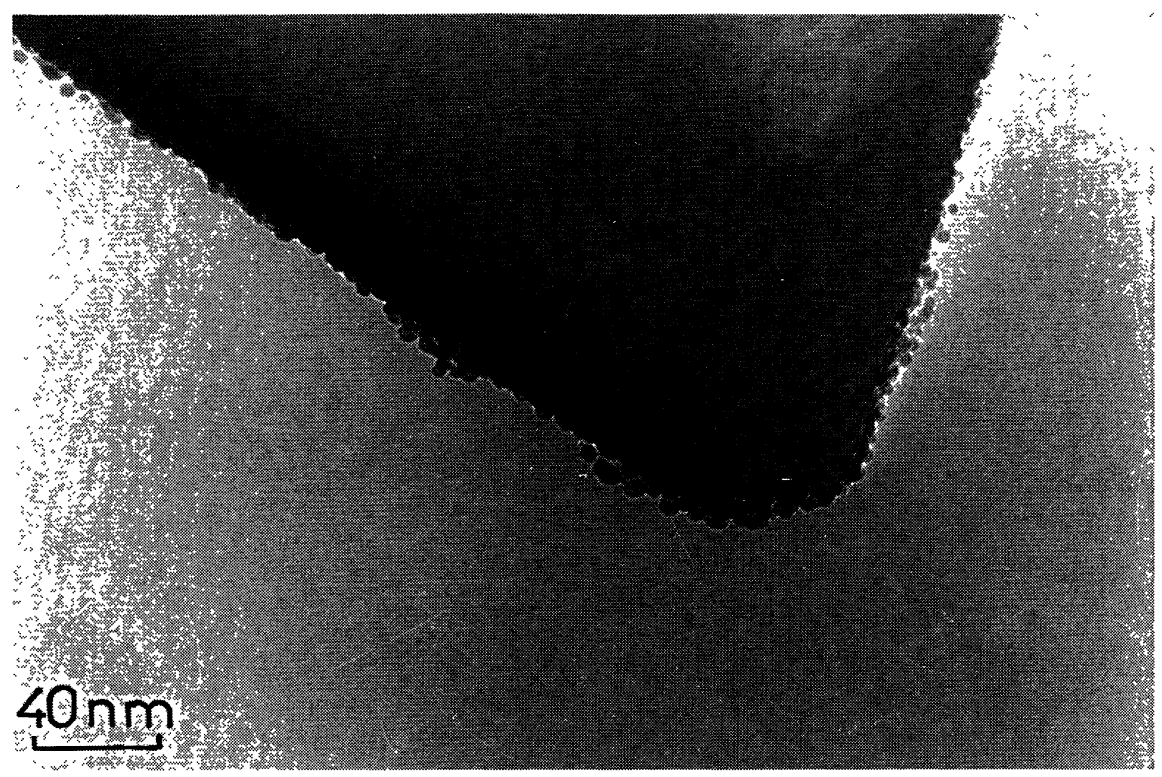

Fig. 7. - Transmission electron microscopy image of a pyramidal AFM tip $\alpha=36^{\circ}$ and $r \sim 40 \mathrm{~nm}$.

Table I shows that a radius variation of $10 \mathrm{~nm}$ induces an area fraction variation of about $3 \%$. The final precision of the area fraction measurement also depends on the precision of the maximum depth $e_{\mathrm{m}}$ and of the experimental measurement of $A_{\mathrm{A}}\left(\gamma^{\prime} e_{\mathrm{m}}\right)$ which are related to the amount of image noise. A relative error of $5 \%$ on $e_{\mathrm{m}}$ and on $A_{\mathrm{A}}\left(\gamma^{\prime} e_{\mathrm{m}}\right)$ gives a relative error on $A_{\mathrm{A}}\left(\gamma^{\prime}\right)$ of $1,5 \%$ and $4 \%$, respectively. The AFM image of figure 1 reveals that the misorientation of the precipitates with respect to the scanning direction is low. Experimentally there is interest in positioning the specimen so that the general orientation of the $\gamma$ channels are parallel to the scanning direction; this is what we have done. Nevertheless, certain precipitates have a small misorientation and this induces a slight overestimation of the $\gamma^{\prime}$ area fraction. Supposing all the precipitates have a misorientation of $\phi=5^{\circ}$, which is certainly less in reality, this would yield an $A_{\mathrm{A}}\left(\gamma^{\prime}\right)$ increase of only $2 \%$.

In the present paper, we have considered the $\gamma^{\prime}$ size distribution as a Dirac function, which is obviously not the case in reality. However, on one hand, it can be noted that the heat treatments currently applied to $\gamma-\gamma^{\prime}$ superalloys lead to very homogeneous precipitate size distribution [9]. On the other hand, the involved etching depths were about ten times smaller than the precipitate mean size. Consequently, the influence of the precipitate size dispersion on the depth 
level histogram is negligible, as compared with the influences of the other bias sources previously discussed.

Finally, since the specimen we studied has a precipitate volume fraction that is known and has been measured to be $69 \%$ by the atom probe chemical analysis [3], we can conclude from our measurements that atomic force microscopy is able, in a relative simple manner, to give an estimate of the precipitate volume fraction of a two-phase Nickel-based superalloy with a precision of a few percent.

\section{Conclusion.}

Measurement of the precipitate volume fraction in Nickel-based superalloys by atomic force microscopy is possible by taking into account the tip dilation effect. The precision of the measurement is mainly determined by the knowledge of the exact shape of the tip. With a classical AFM tip of pyramidal shape the measurement precision is of the order of a few percent. It is possible, by automatisation of the analysis procedure, to get a good estimation of the volume fraction in less than one hour, which is considerably less than with time-consuming techniques such as the atom probe chemical analysis or precipitate chemical extraction and weighing.

\section{Acknowledgements.}

The superalloys single crystals were supplied through the French national research programme "Contrat de Programme de Recherche sur la Stabilité Structurale des Superalliages Monocristallins". The authors wish to thank N. Bonnet for helping and facilitating the image analysis.

\section{References}

[1] Bonnet N., Dongmo S. and Troyon M., Proc. of the 13 rd Cong. of Electron. Microsc. Paris, 1 (1994) 585.

[2] Bonnet N., Dongmo S. and Troyon M., to be published in M.M.M.

[3] Duval S., Chambreland S., Caron P. and Blavette D., Acta Metall. Mater. 42 (1994) 185.

[4] Hazotte A. and Lacaze J., Rev. Métall. CIT (février, 1994) 277.

[5] Georget D. and Peyroutou C., Proc. of the Conf. "High Temperature Materials for Power Engineering 1990”, Liège (Belgium), 24-27 Sept. 90, Bachelet et al. Eds. (Pub. Kluwer Academic Publishers, 1990) p. 1377.

[6] Bourhettar A., Hazotte A. and Troyon M., Proc. of the 33rd Col. of the Société Française de Microscopie Electronique (SFME), Lyon (France, 1993) p. 25.

[7] Bourhettar A., Hazotte A. and Troyon M., submitted to Mater. Charac.

[8] Dehoff R.T. and Rhines F.N., "Quantitative Microscopy” (Mc Graw Hill pub., New-York, U.S.A., 1968).

[9] Caron P. and Khan T., Proc. 1st European Conf. on Advanced Materials and Processes (EUROMAT 89), Aachen nov. 89, H.E. Exner, V. Schumacher Eds. (Publ. D.G.M. Informationgesellschaft Verlag) p. 333.

[10] Vatel O., Thesis, University Aix-Marseille II (France, 1993). 